\title{
ON DENSITY OF ALGEBRAS WITH MINIMAL INVARIANT OPERATOR RANGES
}

\author{
HEYDAR RADJAVI
}

\begin{abstract}
Let $\mathfrak{A}$ be an arbitrary subalgebra of $\mathscr{B}(\mathscr{H})$ and let $\mathscr{N}$ be a dense operator range invariant under $\mathfrak{A}$ such that every nonzero operator range invariant under $\mathfrak{A}$ contains $\mathfrak{R}$. Then the closure of $\mathfrak{A}$ in the strong operator topology is $\mathscr{B}(\mathcal{H})$.
\end{abstract}

Let $\mathfrak{A}$ be a subalgebra of $\mathscr{B}(\mathcal{H})$, the algebra of all (bounded, linear) operators on the complex Hilbert space $\mathcal{H}$. No topological closure assumptions are made on $\mathfrak{A}$. The transitive algebra problem (cf. [7, p. 138]) can be stated as follows: if the only closed subspaces of $\mathcal{H}$ invariant under (all members of) $\mathfrak{A}$ are $\{0\}$ and $\mathcal{H}$, is $\mathfrak{A}$ strongly dense in $\mathscr{B}(\mathcal{H})$ ? Foiaş [5] showed that the answer is affirmative if the hypothesis is strengthened by substituting "operator ranges" for "closed subspaces." By an operator range is meant the range of an operator from $\mathcal{H}$ into $\mathcal{H}$; Foiaş sometimes uses the nomenclature "para-closed subspaces" for operator ranges.

Operator ranges have proven to be useful tools in dealing with questions related to reductivity and reflexivity of operator algebras. The results of Azoff [1] and Douglas and Foiaş [3] are examples. For an extension of the above-quoted result of Foiaş see [6].

In this note we prove strong density for those transitive algebras whose lattice of invariant operator ranges has a simple property; the nonzero elements of the lattice have a nonzero lower bound. Examples of such algebras are abundant; for a simple example let $K$ be an injective operator $\mathcal{H}$ with dense range (but $K \mathcal{H} \neq \mathcal{H}$ to make the example nontrivial). Let $\mathfrak{A}$ be the right ideal $K \mathscr{B}(\mathscr{H})$ of $\mathscr{B}(\mathcal{H})$. Then $K \mathscr{H}$ is certainly invariant under $\mathfrak{A}$ and every nonzero operator range, in fact every nonzero linear manifold, invariant under $\mathfrak{A}$ must include $K \mathfrak{H}$.

We shall need the following lemmas.

LEMMA 1. Let $K$ be an injective operator whose range is a minimal, dense, invariant operator range for the algebra $\mathfrak{A}$. Then there is an algebra isomorphism $\phi$ of $\mathfrak{A}$ into $\mathscr{B}(\mathfrak{H C})$ such that

(i) $A K=K \phi(A)$ for all $A \in \mathfrak{A}$,

(ii) $\phi(\mathfrak{A})$ is strongly dense in $\mathcal{B}(\mathcal{H})$, and

Received by the editors May 27, 1977.

AMS (MOS) subject classifications (1970). Primary 46L15; Secondary 47A15.

Key words and phrases. Invariant subspace, transitive operator algebra, operator ranges.

(c) American Mathematical Society 1978 
(iii) if $K \mathcal{H}$ is contained in all nonzero invariant operator ranges of $\mathfrak{A}$, then the only operators $L$ satisfying the equation $A L=L \phi(A)$ for all $A$ in $\mathfrak{A}$ are scalar multiples of $K$.

Proof. (i) For each $A \in \mathfrak{A}, A K \mathscr{H} \subseteq K \mathcal{H}$ and thus there is an operator $B$ with $A K=K B$; (see, e.g., [2]). The fact that $B$ depends uniquely and isomorphically on $A$ is easy to verify.

(ii) To verify strong density of $\phi(\mathfrak{U})$ it suffices to show that $\phi(\mathfrak{U})$ has no invariant operator ranges other than $\{0\}$ and $\mathcal{H}$ (by Foiaş [5]). Let $M$ be a nonzero operator whose range is invariant under $\phi(\mathfrak{U})$. Assume, with no loss of generality, that $M$ is injective. (If $S$ is an isometry of $\mathcal{H}$ onto $\mathcal{H} / M^{-1}\{0\}$, then $M V$ is injective and has the same range as $M$.) Thus $\phi(A) M=M \psi(A)$ for all $A \in \mathfrak{A}$, where $\psi$ is an algebra homomorphism. Then $A K M=K \phi(A) M$ $=K M \psi(A)$, which implies that $K M \mathcal{H}$ is invariant under $\mathfrak{A}$. By the minimality of $K \mathcal{H}, K \mathcal{H}=K M \mathcal{H}$; since $K$ is injective, we obtain $M \mathcal{H}=\mathcal{H}$.

(iii) First note that $A L=L \phi(A)$ implies that either $L$ is zero or it is injective, because if $L x=0$ with $x \neq 0$, then $L \phi(\mathfrak{U}) x=\mathfrak{A} L x=0$. Since $\phi(\mathfrak{U})$ is strongly dense in $\mathscr{B}(\mathcal{H})$, this implies $L \mathcal{H}=0$.

Now the equation $A L=L \phi(A)$ also implies that $L \mathcal{H}$ is invariant under $\mathfrak{A}$ and thus there is an operator $M$ with $L M=K$. Then

$$
L M \phi(A)=A L M=L \phi(A) M
$$

or, assuming $L \neq 0, M \phi(A)=\phi(A) M$. Since $\phi(\mathfrak{U})$ is dense in $\mathscr{B}(\mathcal{H}), M$ commutes with $\Re(\mathcal{H})$ and must be a scalar operator.

Before stating the next lemma we fix some notation and terminology. For $A \in \mathscr{B}(\mathcal{H})$ we denote by $A^{(n)}$ the direct sum of $n$ copies of $A$ acting on $\mathcal{F}^{(n)}$, the direct sum of $n$ copies of $\mathcal{H}$; for an algebra $\mathfrak{A}$ of operators, $\mathfrak{A}^{(n)}$ will stand for $\left\{A^{(n)}: A \in \mathfrak{A}\right\}$. A graph subspace for $\mathfrak{H}^{(n)}$ is a closed subspace of $\mathcal{H}^{(n)}$, invariant under $\mathfrak{I}^{(n)}$, which has the form

$$
\left\{x \oplus T_{1} x \oplus \cdots \oplus T_{n-1} x: x \in \mathscr{D}\right\},
$$

where $\mathscr{D}$ is a dense linear manifold in $\mathcal{H}$ and, for each $i, T_{i}$ is a linear transformation from $\mathscr{D}$ into $\mathcal{H}$. Each $T_{i}$ is called a graph transformation for $\mathfrak{U}$. This definition implies, in particular, that $\mathscr{D}$ and the $T_{i} \mathscr{D}$ are invariant under $\mathfrak{A}$.

The following lemma due to Arveson, will be used; for a proof see [7, $p$. 143].

LEMMA 2. Let $\mathfrak{A}$ be an algebra with the property that every graph transformation for $\mathfrak{A}$ is a scalar multiple of the identity on $\mathcal{H}$. Then $\mathfrak{A}$ is strongly dense in $\mathscr{B}(\mathcal{H})$.

(Note that the hypothesis on $\mathfrak{A}$ implies that $\mathfrak{A}$ has no closed invariant subspaces except $\{0\}$ and $\mathcal{H}$.)

TheOrem. Let $\mathfrak{A}$ be a subalgebra of $\mathscr{B}(\mathcal{H})$. Assume that $\mathfrak{U}$ has a dense, 
invariant operator range contained in every nonzero invariant operator range. Then $\mathfrak{A}$ is strongly dense in $\mathscr{B}(\mathcal{H})$.

Proof. Let $K \in \mathscr{B}(\mathcal{H})$ such that $K \mathcal{H}$ is the lower bound of the nonzero invariant operator ranges as hypothesized. The operator $K$ can also be assumed to be injective. Now $A K=K \phi(A)$ for all $A \in \mathfrak{A}$, where $\phi$ is the isomorphism given by Lemma 1.

Let $n$ be any positive integer and let $\mathscr{T}$ be a graph subspace for $\mathfrak{U}^{(n)}$. Then $T$ can be considered as the graph of a closed linear transformation $T$ from a dense domain $\mathscr{D}$ in $\mathcal{H}$ into $\mathcal{H}^{(n-1)}$ defined by $T x=T_{1} x \oplus \cdots \oplus T_{n-1} x$, $x \in \mathscr{D}$. Now $\mathscr{D}=C_{1} \mathcal{H}$, where $C_{1}$ is a bounded, injective operator on $\mathcal{H}$ (see, e.g., [4]). Thus

$$
\mathfrak{K}=\left\{C_{1} y \oplus T C_{1} y: y \in \mathscr{H}\right\}
$$

since $T C_{1}$ is closed, it follows from the closed-graph theorem that it is bounded. Hence $T_{i} C$ is bounded, for each $i$, because $T_{i} C=P_{i} T C$, where $P_{i}$ is a projection. We can now represent $\mathfrak{N}$ as

$$
\left\{C_{1} x \oplus C_{2} x \oplus \cdots \oplus C_{n} x: x \in \mathcal{H}\right\},
$$

where each $C_{i}$ is a bounded operator and where $C_{1}$ is, furthermore, injective.

The inclusion $A^{(n)} \mathfrak{R} \subseteq \mathfrak{R}$ implies that for each $x$ and each $A \in \mathfrak{A}$ there exists a $y$ with $A C_{i} x=C_{i} y$ for $i=1, \ldots, n$. The injectivity of $C_{1}$ implies that $y$ is unique, and it follows that there is an algebra isomorphism $\psi$ of $\mathfrak{A}$ into $\mathscr{B}(\mathcal{H})$ such that $A C_{i}=C_{i} \psi(A)$ for all $A$ and all $i$. In particular, $C_{1} \mathscr{H}$ is invariant under $\mathfrak{A}$ and thus $C_{1} B=K$ for some operator $B$. Thus, by the equation $A K=K \phi(A)$,

$$
C_{1} B \phi(A)=A C_{1} B=C_{1} \psi(A) B
$$

and, therefore, $B \phi(A)=\psi(A) B$. Now, for each $i$,

$$
A C_{i} B=C_{i} \psi(A) B=C_{i} B \phi(A),
$$

and Lemma 1 implies that $C_{i} B=\alpha_{i} K=\alpha_{i} C_{1} B$ for some scalar $\alpha_{i}$. Thus

$$
y \oplus \alpha_{2} y \oplus \cdots \oplus \alpha_{n} y \in \Re
$$

for every $y \in K \mathcal{H}$. Since $K \mathcal{H}$ is dense in $\mathcal{H}$,

$$
\mathfrak{K}=\left\{y \oplus \alpha_{2} y \oplus \cdots \oplus \alpha_{n} y: y \in \mathcal{H}\right\} .
$$

We have shown that every $T_{i}$ is a scalar multiple of the identity on $\mathcal{H}$. The strong density of $\mathfrak{A}$ in $\mathscr{B}(\mathcal{H})$ now follows from Lemma 2.

COROLlaRY 1. If in the lattice of invariant operator ranges for $\mathfrak{A}$ the nonzero elements have a nonzero lower bound, then either $\mathfrak{A}$ is strongly dense in $\mathscr{B}(\mathcal{H})$ or it has a nontrivial closed, invariant subspace.

Proof. If this lower bound represents a dense range, we apply the above theorem; otherwise, the closure of this range is a nontrivial invariant subspace.

Corollary 2. If $\mathfrak{A}$ is as in Corollary 1 and if $\mathfrak{M}$ is the lower bound for 
nonzero, invariant operator ranges, then the restriction $\mathfrak{A} \mid \overline{\mathfrak{N}}$ of $\mathfrak{A}$ of the closure of $\Re$ is dense in $\mathscr{B}(\bar{\Re})$.

Proof. It is easily verified that $\mathscr{N}$ is the lower bound of the nonzero operator ranges invariant under $\mathfrak{A} \mid \overline{\mathfrak{T}}$; thus the theorem is applicable.

Questions. One natural question poses itself: Can the lattice-theoretic hypothesis of the above results be weakened to require the mere existence of a minimal nonzero element in this lattice? What about the more special case where every nonzero member of the lattice is assumed to contain a minimal nonzero element?

\section{REFERENCES}

1. Edward A. Azoff, Invariant linear manifold and the self-adjointness of operator algebras, Amer. J. Math. 99 (1977), 121-137.

2. R. G. Douglas, On majorization, factorization, and range inclusion of operators on Hilbert space, Proc. Amer. Math. Soc. 17 (1966), 413-415.

3. R. G. Douglas and C. Foias, Infinite dimensional versions of a theorem of Brickman-Fillmore, Indiana Univ. Math. J. 25 (1976), 315-320.

4. P. A. Fillmore and J. P. Williams, On operator ranges, Advances in Math. 7 (1971), 254-281.

5. C. Foias, Invariant para-closed subspaces, Indiana Univ. Math. J. 21 (1972), 887-906.

6. E. Nordgren, H. Radjavi and P. Rosenthal, Operator algebras leaving compact operator ranges invariant, Michigan Math. J. 23 (1976), 375-377.

7. H. Radjavi and P. Rosenthal, Invariant subspaces, Springer-Verlag, Berlin and New York, 1973.

Department of Mathematics, Dalhousie University, Halifax, Nova Scotia, Canada 